\title{
La diferenciación de áreas de mercado laboral juvenil homogéneas, en el municipio de Getafe
}

\author{
José Miguel Santos Preciado
}

\begin{abstract}
RESUMEN ABSTRACT
La aplicación de técnicas

The application of classification techniques (using the factorial model) clasificatorias (utilizando el modelo factorial) permite identificar áreas homogéneas de mercado de trabajo juvenil, en el municipio de Getafe, mediante el empleo de variables sociodemográficas, formativas $y$ de estructura profesional, relacionadas con la segmentación y dualización

laboral actualmente existente. El carácter discriminador del territorio sirve de marco de referencia, para comprender la génesis de este tipo

de áreas, como resultado de los procesos de segregación espacial,

que están teniendo lugar en el

citado municipio, desde los años

sesenta. leads to the identification of homogeneous areas in the market of employment for the young people in the municipality of Getafe. This is achieved using variables for sociodemography, education, and professional structure, related to the segmentation and dualitazion of labour which currently exist. The discrimination nature of the district acts as a frame of reference to understand the origin of this type of area, as resulting from the processes of spatial segregation which have been taking place since the decade of 1960 s.
\end{abstract}

\section{INTRODUCCIÓN}

Quizás, la variable más definitiva de todas, en el intento de construir modelos teóricos de la problemática laboral juvenil, resulte ser el origen social. Esta variable mantiene una estrecha correlación con el territorio, 
hasta el punto de que tenga sentido referirse a la existencia de un factor socio-territorial a la cabeza de los condicionamientos del mundo del trabajo. La homogeneidad de la estructura social de los barrios donde habitan los jóvenes es decisiva en su comportamiento, de cara a la inserción en el mercado laboral. Como si de un continuo social se tratara, encontraríamos en un extremo de la escala a los barrios marginales, habitados por inmigrantes sin cualificación ni estudios, y en el opuesto a los barrios residenciales de clase media alta o elevada, lugares de residencia de empresarios y profesionales de elevada cualificación y alto nivel de ingresos. Un análisis de las situaciones profesionales de los jóvenes, que surgen de ambientes sociales contrastados, nos serviría de pauta para definir las problemáticas tipo de su integración laboral.

En este sentido, los mercados laborales juveniles no pueden ser concebidos de forma unitaria. Cabe, al menos, referirse a la existencia de un mercado primario, caracterizado por una mano de obra, con elevado nivel de educación y alta cualificación profesional, frente a un mercado secundario, integrado por trabajadores de bajo nivel de estudios, escasa formación ocupacional, que realiza los trabajos menos cualificados. Esta segmentación del mercado laboral juvenil ha tenido diferente incidencia, según zonas de la región urbana madrileña, originando espacios sociales, laboralmente uniformes, en lo que respecta a la problemática de su incorporación al mundo del trabajo (Mayoral Lobato, J.; 1992).

De las diversas líneas teóricas que iluminan el concepto de mercado laboral, el relativo a la estructura productiva nos parece el más fecundo. Esta línea de investigación permite establecer una certera conexión entre la problemática socio-laboral y su articulación geográfica o territorial. Por una parte, la organización empresarial (estructura de la oferta) demanda un tipo determinado de fuerza de trabajo (según coste, cualificación, etc.), mientras que por otra, la estructura de la población activa (estructura de la demanda) genera impactos y determinaciones de gran alcance sobre la organización de la industria y los servicios. Es, precisamente, este segundo componente, el de la caracterización del factor trabajo, el que permite valorar, a nivel municipal, los desequilibrios territoriales generados en el espacio. Por este motivo, utilizaremos la técnica de la regionalización como instrumento capaz de relacionar la problemática de la incorporación de los jóvenes al mundo laboral con el territorio. Será, por tanto, la estructura de la población activa laboral la referencia de medida de la diferenciación espacial de áreas de mercado de trabajo homogéneas. 


\section{LA DIFERENCIACIÓN DE ÁREAS DE MERCADO LABORAL JUVENIL HOMOGÉNEAS, MEDIANTE LA TÉCNICA FACTORIAL}

La regionalización y su metodología consecuente ha ocupado, desde siempre, una posición central entre las preocupaciones geográficas, como una forma lógica y satisfactoria de organizar la información geográfica. En este sentido, la regionalización homogénea, como instrumento de delimitar el espacio en áreas uniformes, respecto a un fenómeno o hecho concreto, no es sino un apartado de la clasificación, procedimiento de análisis que pretende organizar los elementos, objeto de estudio de una determinada materia, en un conjunto de categorías más reducido, según unos criterios fijados previamente. En el caso de la regionalización homogénea, a la similitud de los elementos de cada clase resultante se debe añadir, que las unidades que pertenecen a una región deben tener una contiguidad espacial.

Definida la regionalización homogénea como una técnica clasificatoria particular, debemos exponer que su consecución consta de varias fases diferenciadas (Santos Preciado, J.M.; 1992) (figura 1). El problema de determinar las propiedades en que se basa la clasificación es de naturaleza científica y supone la selección previa de una matriz de datos, relacionada con los objetos a clasificar y los fenómenos que orientan la clasificación. En nuestro caso, las secciones censales del municipio de Getafe (94 unidades geográficas) (figura 2), como elementos espaciales unitarios, de carácter administrativo, que albergan la población joven, serán los objetos de la clasificación, mientras la estructura socioprofesicnal y formativa de la población activa juvenil, definida por un conjunto de variables, que expondremos a continuación, constituirá la referencia de medida de la misma.

Esta matriz rectangular puede ser transformada con objeto de obtener las dimensiones fundamentales de las variables seleccionadas. De todas las técnicas de sintetizar la información, posiblemente, la técnica factorial sea la más conocida y utilizada. Al igual que otras muchas técnicas matemáticas, el análisis factorial (AF) constituye un modelo que trata de reproducir lo mejor posible el mundo real, es decir, se presume que la estructura de éste es isomórfica con una determinada formulación matemática (Bosque Sendra, J. y Moreno Jiménez, A.; 1994). El AF es, en realidad, un método estadístico que trata de sintetizar la información en un número reducido de factores. En nuestro caso, la elevada correlación existente entre las variables va a concentrar la información de partida en un único factor. 


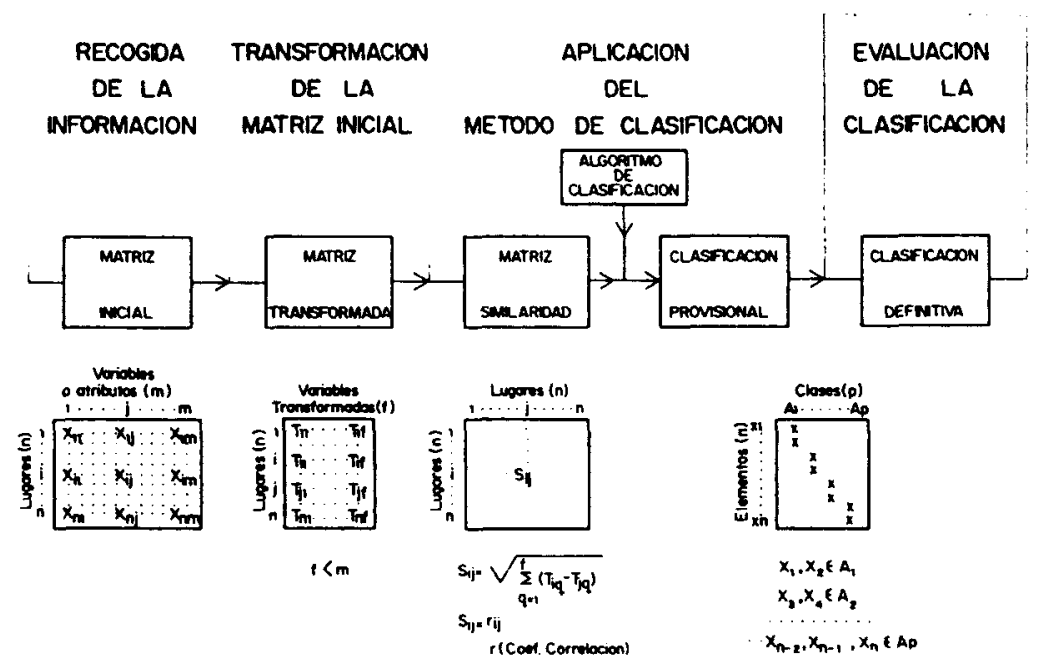

Figura 1. Metodología de la clasificación.

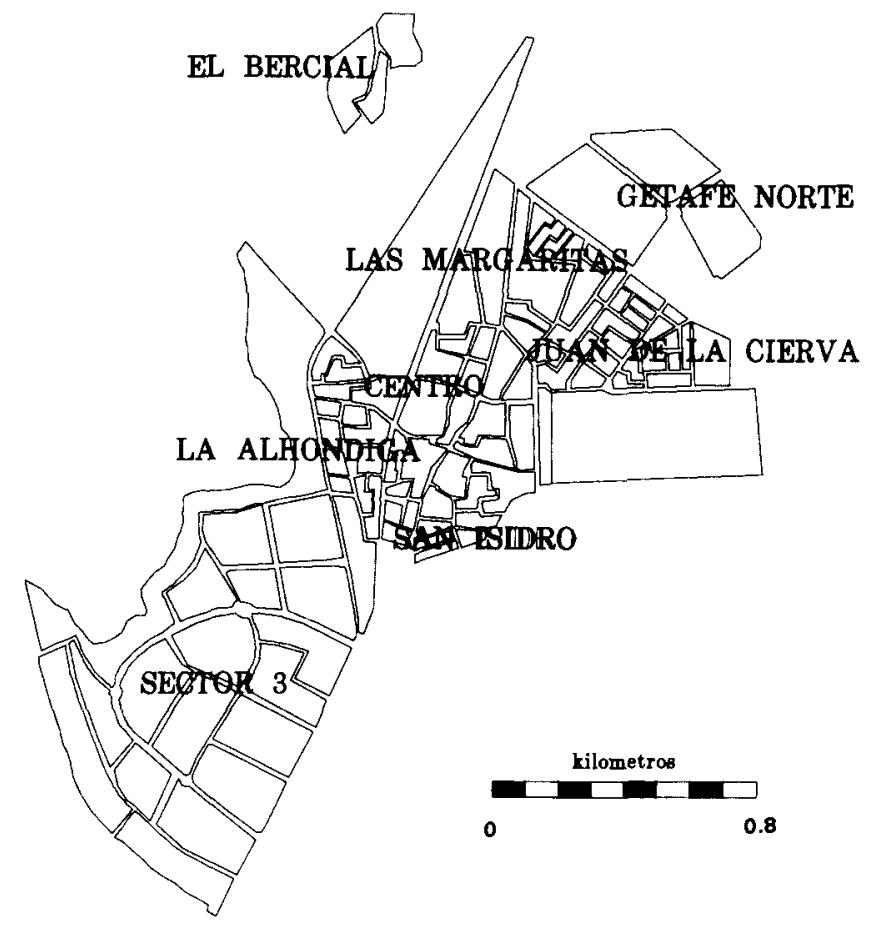

Figura 2. Secciones censales (por barrios) del municipio de Getafe. 
El siguiente paso del método taxonómico consiste en definir una medida de similaridad, con objeto de obtener el nivel de semejanza de los individuos u objetos del análisis. En general, esta fase del proceso supone la comparación de los elementos entre sí, de acuerdo a un criterio o atributo simple o múltiple. La riqueza de los métodos clasificatorios estriba en la gran cantidad de procedimientos existentes para realizar esta operación particular. En el caso que nos ocupa, la existencia de un solo factor que sintetiza la información simplifica la operación, ya que la comparación de los elementos del análisis se realiza por las notaciones factoriales (valor del primer factor en cada una de las secciones censales).

\subsection{La aplicación de la técnica factorial al estudio de la delimitación de áreas de mercado juveniles homogéneas.}

La aplicación del modelo factorial exige la consecución de cuatro fases perfectamente diferenciadas (Santos Preciado, J.M.; 1991):

- Preparación del material empírico, base de la investigación.

- Factorización (obtención de la matriz factorial).

- Rotación (obtención de la matriz factorial rotada).

- Interpretación de los resultados.

La definición de las variables, representativas de la problemática laboral juvenil unitaria, nos ha venido facilitada por un estudio previo que hemos realizado, relativo a la incorporación de los jóvenes del A.M.M. al mercado laboral (Aguilera Arilla y otros; 1996). La dualización de la estructura ocupacional, derivada de la fragmentación del mercado de trabajo juvenil en segmentos muy contrastados (un mercado primario de empleos primarios, de alto nivel de cualificación bien pagados y con amplias posibilidades de promoción profesional y un mercado secundario de empleos inestables, de perfil profesional inferior, mal pagados y con escasas posibilidades de superación personal), allana el camino hacia la definición de un conjunto de variables, de diferente carácter, formativo y laboral, que sirven de vínculo de relación con el territorio.

Estas variables seleccionadas han sido las siguientes:

nivel formativo

- tasa de escolaridad (población de 20 a 29 años).

- tasa de estudios de tercer grado universitario (población de 25 a 29 años). 


\section{relación con la actividad}

- tasa de actividad masculina (población de 15 a 24 años).

- tasa de actividad femenina (población de 25 a 29 años).

relación con los modos de producción

- porcentaje de trabajadores fijos sobre el total de los activos.

ramas productivas

- porcentaje de trabajadores que trabajan en la Construcción, respecto al total de la población activa.

- porcentaje de trabajadores que trabajan en la rama de Educación, respecto al total de la población activa.

\section{estructura profesional} activa.

- porcentaje de técnicos superiores, respecto al total de la población

- porcentaje de técnicos medios e inferiores, respecto al total de la población activa.

- porcentaje de trabajadores no cualificados, respecto al total de la población activa.

Aplicado el modelo de los factores principales, que diferencia los factores principales, aquellos que están relacionados al menos con dos o más variables, de los específicos, únicamente relacionados con cada variable, los porcentajes de varianza explicados por cada factor vienen expresados en el Cuadro I. Como puede observarse, el primer factor concentra el $75.4 \%$ de la varianza, lo que significa que sintetiza más de las tres cuartas partes de la información.

Cuadro 1. Porcentaje de la varianza, explicado por cada factor.

\begin{tabular}{ccc}
\hline factor & varianza explicada & Varianza acumulada \\
\hline 1 & $75.4 \%$ & $75.4 \%$ \\
2 & $12.8 \%$ & $88.2 \%$ \\
3 & $5.2 \%$ & $93.4 \%$ \\
4 & $3.5 \%$ & $96.9 \%$ \\
5 & $1.6 \%$ & $98.5 \%$ \\
6 & $1.5 \%$ & $100.0 \%$ \\
7 & $0.0 \%$ & $100.0 \%$ \\
8 & $0.0 \%$ & $100.0 \%$ \\
9 & $0.0 \%$ & $100.0 \%$ \\
10 & $0.0 \%$ & $100.0 \%$ \\
\hline
\end{tabular}


El significado de este factor puede deducirse de la matriz factorial (Cuadro II) (relativa, en este caso a los dos primeros factores, aunque el que realmente nos interesa es el primero de ellos). La correlación positiva del primer factor con las variables: técnicos de nivel superior, medio e inferior y negativa con el porcentaje de trabajadores no cualificados supone la oposición, lógica, de categorías profesionales contrastadas y atribuye al mismo una relación de oposición entre mercados laborales extremos. Esta realidad se ve corroborada por la antítesis existente entre otras variables: tasa de actividad masculina y femenina y trabajadores de la construcción frente a trabajadores fijos y de la rama de Educación. Además, en el mismo sentido, se correlacionan, positivamente, la tasa de escolaridad y la tasa de titulados universitarios.

En resumen, se podría expresar la existencia de dos tipos de mercados laborales contrastados, definidos cada uno de ellos por su correlación con las siguientes variables:

\section{mercado primario}

- alta tasa de escolaridad, abandono tardío del sistema escolar.

- alta tasa de titulados universitarios, que evidencia un nivel de estudios elevado.

- alta tasa de actividad de la mujer mayor de 25 años, que se corresponde con un mayor porcentaje de mujeres casadas que trabajan.

- menor tasa de actividad masculina de los jóvenes menores de 24 años, que supone una integración más tardía en el mundo del trabajo.

- un pertil ocupacional de mayor rango, relacionado con profesionales técnicos, más cualificados, que trabajan en ramas productivas como la Educación (ocupada, en gran parte por mujeres de mayor nivel social).

Cuadro II. Matriz factorial.

\begin{tabular}{lrr}
\hline \multicolumn{1}{c}{ variables } & factor 1 & factor 2 \\
\hline Tasa de escolaridad & 0.8520 & 0.1278 \\
Tasa de estudios universitarios & 0.8384 & -0.1866 \\
Tasa de actividad masculina & -0.8712 & -0.2221 \\
Tasa de actividad femenina & 0.3704 & -0.1831 \\
Trabajadores fijos & 0.3100 & 0.0949 \\
Trabajadores de la Construcción & -0.5950 & -0.0729 \\
Trabajadores en Educación & 0.4058 & -0.5084 \\
Técnicos superiores & 0.7146 & -0.4376 \\
Técnicos medios e inferiores & 0.7217 & 0.2639 \\
Trabajadores no cualificados & -0.6468 & -0.2772 \\
\hline
\end{tabular}




\section{mercado secundario} reglado.

- baja tasa de escolaridad, abandono temprano del sistema educativo

- baja tasa de estudios universitarios, índice correlativo de menor nivel de estudios.

- baja tasa de actividad femenina de la mujer mayor de 25 años, que se corresponde con un mayor porcentaje de mujeres casadas que no trabajan.

- mayor tasa de actividad masculina de jóvenes menores de 24 años, que indica una incorporación más temprana al mercado de trabajo.

- un perfil ocupacional de menor rango, definido por la relación con los trabajadores no cualificados y de la construcción.

La cartografía de las notaciones factoriales del primer factor (figura 3) revela la existencia de zonas, que albergan estructuras sociolaborales juveniles muy contrastadas. Esta realidad pone de manifiesto, de forma sintética, tendencias que ya habían aparecido, individualmente, para cada una de las variables más representativas del espectro profesional y formativo de los jóvenes. A modo de resumen, podríamos calificar el carácter de los barrios del municipio de Getafe:

- Dos áreas homogéneas, de superior nivel relativo, en lo que respecta al rango social y a los rasgos propios a un mercado de trabajo primario. Ambas coinciden, casi plenamente con los barrios del Sector 3 y Getafe Norte.

- Tres áreas homogéneas, de menor categoría social, coincidentes, en gran parte con la naturaleza del mercado laboral secundario. Estas áreas se corresponden con los barrios de La Alhóndiga, San Isidro y Las Margaritas.

- Dos zonas heterogéneas, de nivel medio-bajo, coincidentes con los barrios de Juan de la Cierva y El Bercial.

- Un área heterogénea, con fuertes contrastes geográficos internos, que se corresponde con el casco antiguo o barrio Centro del municipio.

\section{LA INTERPRETACIÓN DE LOS RESULTADOS}

La diferenciación de las características socioprofesionales de los jóvenes de Getafe no es sino la consecuencia del proceso de desarrollo urbano, que ha tenido lugar en el municipio, de forma acelerada, desde los 


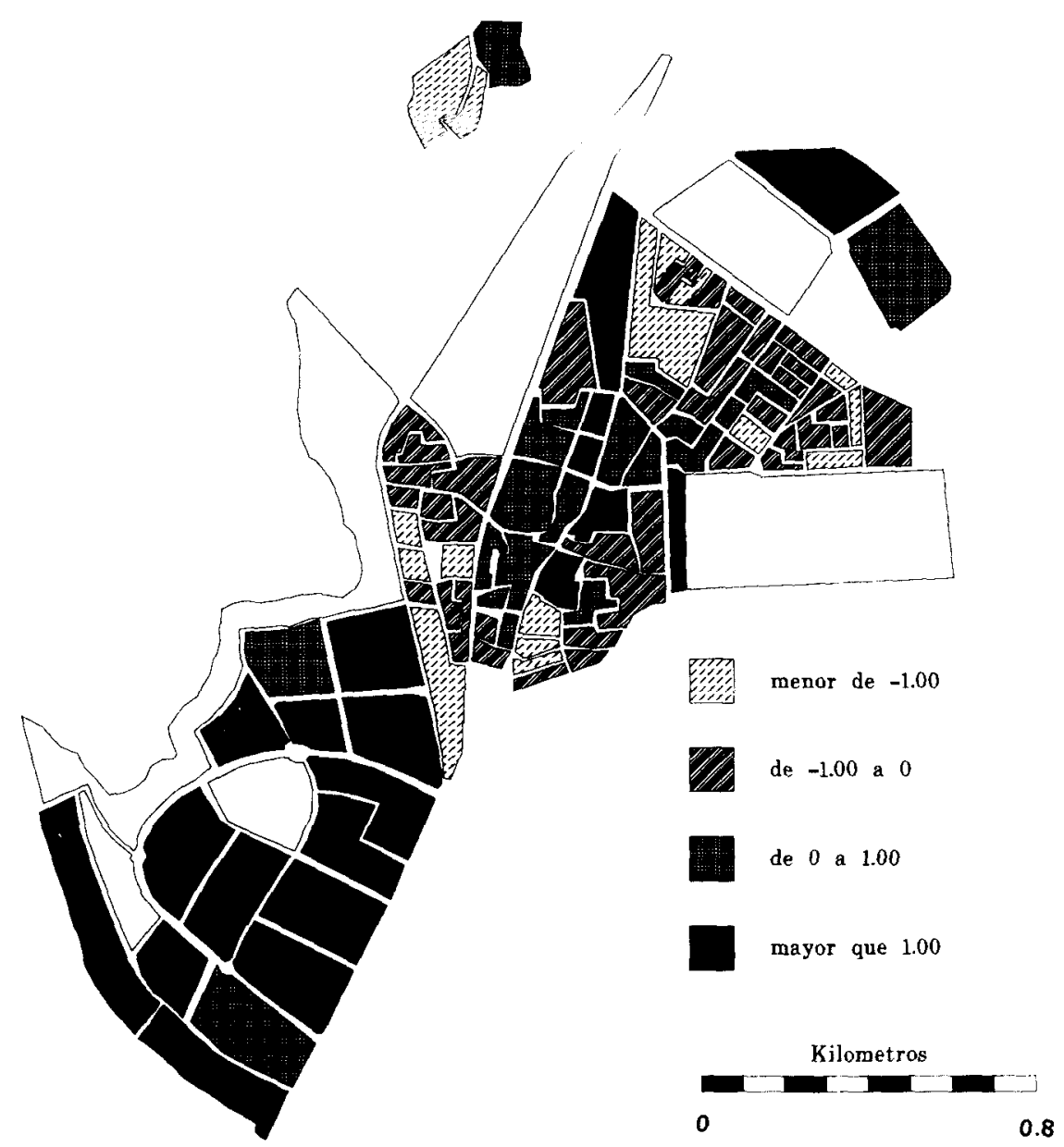

Figura 3. Notaciones factoriales del primer factor del AF.

primeros años sesenta. Este proceso, claramente segregativo en el espacio y en el tiempo, ha perfilado la identidad de cada barrio, en consonancia con el modelo de diferenciación residencial que ha afectado a toda el área metropolitana madrileña (Santos Preciado, J.M.; 1989),

Fue, precisamente, a partir de estos años, cuando se verificó un cambio profundo en el modelo de desarrollo urbano del hinterland madrileño, fruto del crecimiento económico, experimentado por el país, que reestructuró, territorialmente, los municipios próximos a la ciudad de Madrid. Las 
particulares circunstancias urbanísticas que concurrían en el municipio de Getafe (proximidad a la capital, temprana localización de la industria y de otras funciones urbanas, excelente situación respecto a las carreteras de salida de Madrid y ferrocarril, etc.) explican que tuviera, en 1960, un peso poblacional muy importante, de unos veinte mil habitantes. Pero fue, durante los años sesenta y comienzo de la década siguiente, cuando el crecimiento de Getafe resultó más espectacular. La búsqueda, por parte de los promotores inmobiliarios, de suelo abundante y barato, donde localizar la demanda de vivienda de la población inmigrante que acudía masivamente a la capital, determinó una expansión urbana sin precedentes, que alcanzó, igualmente, a otros municipios de la primera corona metropolitana, como Alcorcón o Leganés. La población llegó a multiplicarse por cinco, en menos de una década, superando la cifra de los cien mil habitantes.

La mayor parte de las nuevas viviendas se construyeron en los nuevos ensanches residenciales de Juan de la Cierva, Las Margaritas, San Isidro y La Alhóndiga, que, en continuidad con el tejido urbano anterior, albergaron gran parte de las iniciativas de los promotores de la vivienda. Surgen, igualmente, por estas fechas los barrios de El Bercial y Perales del Río, alejados del casco urbano municipal y la promoción residencial en el casco antiguo, de relativa extensión, donde llegaron a edificarse casi el $20 \%$ de las viviendas de nueva construcción. La permisividad de aquellos años, general, por otra parte, en el panorama urbanistico español, originó graves deficiencias de urbanización y construcción, con ausencia de equipamientos de todo tipo. El carácter social del municipio era, en general, bajo, salvo áreas concretas del centro urbano, vertebrado a lo largo de la calle Madrid, que, en realidad, no hacía sino continuar el rango social de etapas anteriores y determinadas zonas de algunos barrios, como el de Juan de la Cierva, donde se produjeron promociones de viviendas, de menor tamaño, dirigidas a clases sociales de mayor nivel de renta relativo.

El frenazo al crecimiento urbano, que tuvo lugar desde casi la mitad de la década de los años setenta, fue la consecuencia de dos circunstancias que afectaron al municipio de forma paralela: la incidencia de la crisis económica, que ralentizó el desarrollo anterior, y el salto de la promoción de vivienda barata a los municipios de la segunda corona metropolitana (Mostoles, Parla y Fuenlabrada). Este proceso se modificó con la llegada de los ayuntamientos democráticos y la aprobación del plan del Sector 3, que a partir de 1978 desarrolló un movimiento cooperativista de construcción de viviendas unifamiliares, sobre un área de más de $300 \mathrm{Has}$, prácticamente el mismo espacio ocupado por el nucleo urbano edificado hasta entonces. Este área contrasta, socialmente, con resto del municipio, al albergar una población más joven y de mayor nivel socioeconómico. 
Durante los últimos años, las nuevas tendencias de localización residencial, promovidas, en parte, desde el propio ayuntamiento, con el fomento de viviendas protegidas, que ofrecieran a los jóvenes la posibilidad de permanecer en el propio municipio, así como otras, dirigidas a capas de población de superior nivel de renta relativa, ha desarrollado el barrio de Getafe Norte, que recoge la mayor parte del reciente crecimento residencial del municipio.

Este proceso de desarrollo urbano, sumariamente descrito, explica las diferentes áreas sociales existentes en el municipio de Getafe, que reproducen, igualmente, diferencias socioterritoriales de los colectivos de jóvenes que en ellas habitan.

\section{BIBLIOGRAFIA}

Aguilera ARILla, M.J. y otros (1996): Desequilibrios del mercado de trabajo juvenil en el A.M.M. Proyecto de investigación, subvencionado por la Oficina Madrileña de Fomento a la Investigación. Consejeria de Educación y Cultura de la C.A.M. Madrid, 527 págs.

Bosque Sendra, J. y Moreno Jimenez. A. (1994): Prácticas de análisis exploratorio y multivariante de datos. Ed. Oikos Tau. Barcelona. 214 págs.

CASAL BAtAlleR, J. y otros (1991): La inserción social y profesional de los jóvenes. Ministerio de Educación y Ciencia. Madrid.

EQUIPO EUSYA (1988): Paro y empleo juvenil en la periferia urbana madrileña. Consorcio Rector del Plan de Prevención de la Delincuencia y la Marginación Social en San Blas, Orcasitas y Pan Bendito. Madrid.

Mayoral Lobato, J. (1992): Mercado de trabajo, políticas de empleo y desarrollo local. Fundación Universidad Empresa. Irmesa. Madrid, 375 págs.

MONTORO, R. (1985): La inserción en la actividad económica: empleo y paro juvenil. Publicaciones de Juventud y Sociedad S.A. Instituto de la Juventud. Ministerio de Cultura. Madrid, 191 págs.

O.C.D.E./C.E.R.I. (1989): Inserción de los jóvenes en una sociedad en cambio. Ed. Narcea. Madrid, 167 págs.

Santos Preciado, J.M. y otros (1987): "Los mercados locales de empleo". Alfoz. Octubre. págs. 30-49. Madrid.

SANTOS PRECIADO, J.M. (1989):El modelo de diferenciación residencial del sector suroeste del A.M.M. Ed. Universidad Complutense de Madrid. Madrid, 1045 págs.

SANTOS PRECIADO, J.M. (1991): "La técnica factorial y su aplicación al campo geogrático". Espacio, Tiempo y Forma. UNED. Madrid, págs. 13-78.

SANTOS PRECIADO, J.M. (1992): “La aplicación de la metodología y técnicas de clasificación en la jerarquización de los asentamientos urbanos de un territorio. Breve repaso a alguno de los principales trabajos de la investigación geográfica española". Actas V Coloquio de Geografia Cuantitativa. Zaragoza. págs. 559-577.

Sauvage, P. (1988): Insertion des jeunes et modernization. Ed. Económica. Paris, 204 págs. 